\title{
THE NASA-UC ETA-EARTH PROGRAM. III. A SUPER-EARTH ORBITING HD 97658 AND A NEPTUNE-MASS PLANET ORBITING G1 785*
}

\author{
Andrew W. Howard ${ }^{1,2}$, John Asher Johnson ${ }^{3}$, Geoffrey W. Marcy ${ }^{1}$, Debra A. Fischer ${ }^{4}$, Jason T. Wright ${ }^{5,6}$, \\ Gregory W. Henry ${ }^{7}$, Howard Isaacson ${ }^{1}$, JefF A. Valenti ${ }^{8}$, Jay Anderson ${ }^{8}$, and Nikolai E. Piskunov ${ }^{9}$ \\ ${ }^{1}$ Department of Astronomy, University of California, Berkeley, CA 94720-3411, USA \\ ${ }^{2}$ Space Sciences Laboratory, University of California, Berkeley, CA 94720-7450 USA; howard@astro.berkeley.edu \\ ${ }^{3}$ Department of Astrophysics, California Institute of Technology, MC 249-17, Pasadena, CA 91125, USA \\ ${ }^{4}$ Department of Astronomy, Yale University, New Haven, CT 06511, USA \\ ${ }^{5}$ Department of Astronomy and Astrophysics, The Pennsylvania State University, University Park, PA 16802, USA \\ ${ }^{6}$ Center for Exoplanets and Habitable Worlds, The Pennsylvania State University, University Park, PA 16802, USA \\ ${ }^{7}$ Center of Excellence in Information Systems, Tennessee State University, 3500 John A. Merritt Blvd., Box 9501, Nashville, TN 37209, USA \\ ${ }^{8}$ Space Telescope Science Institute, 3700 San Martin Dr., Baltimore, MD 21218, USA \\ ${ }^{9}$ Department of Astronomy and Space Physics, Uppsala University, Box 515, 75120 Uppsala, Sweden \\ Received 2010 November 2; accepted 2010 December 30; published 2011 February 24
}

\begin{abstract}
We report the discovery of planets orbiting two bright, nearby early K dwarf stars, HD 97658 and G1 785. These planets were detected by Keplerian modeling of radial velocities measured with Keck-HIRES for the NASA-UC Eta-Earth Survey. HD $97658 \mathrm{~b}$ is a close-in super-Earth with minimum mass $M \sin i=8.2 \pm 1.2 M_{\oplus}$, orbital period $P=9.494 \pm 0.005$ days, and an orbit that is consistent with circular. Gl $785 \mathrm{~b}$ is a Neptune-mass planet with $M \sin i=21.6 \pm 2.0 M_{\oplus}, P=74.39 \pm 0.12$ days, and orbital eccentricity $e=0.30 \pm 0.09$. Photometric observations with the T12 $0.8 \mathrm{~m}$ automatic photometric telescope at Fairborn Observatory show that HD 97658 is photometrically constant at the radial velocity period to $0.09 \mathrm{mmag}$, supporting the existence of the planet.
\end{abstract}

Key words: planetary systems - stars: individual (HD 97658, G1 785) - techniques: radial velocities

\section{INTRODUCTION}

Radial velocity (RV) searches for extrasolar planets are discovering less massive planets by taking advantage of improved instrumental precision, higher observational cadence, and diagnostics to identify spurious signals. These discoveries include planets with minimum masses $(M \sin i)$ as low as $1.9 M_{\oplus}$ (Mayor et al. 2009) and systems of multiple low-mass planets (e.g., Lovis et al. 2006; Fischer et al. 2008; Vogt et al. 2010). To date, 15 planets with $M \sin i<10 M_{\oplus}$ and 18 planets with $M \sin i=10-30 M_{\oplus}$ have been discovered by the RV technique (Wright et al. 2010; Exoplanet Orbit Database ${ }^{10}$ ). Transiting searches for extrasolar planets have detected Neptune-mass planets (Bakos et al. 2010; Hartman et al. 2010) and super-Earths (Léger et al. 2009; Charbonneau et al. 2009). The initial data release from the Kepler mission shows substantially increasing planet occurrence with decreasing planet radius (Borucki et al. 2010). Using the large number of low-mass planets, we can statistically study planet properties, occurrence rates, and parameter correlations in ways previously only possible with higher mass gas-giant planets.

The distribution of low-mass planets in mass and orbital distance offers a critical probe of the mechanisms of planet formation. Howard et al. (2010b, hereafter H10) analyzed the rate of close-in planet occurrence for $166 \mathrm{G}$ - and K-type dwarf stars in the Eta-Earth Survey. H10 measured rapidly increasing planet occurrence with decreasing planet mass over the planet mass range 3-1000 $M_{\oplus}$ for orbital periods $P<50$ days. For close-in super-Earths and Neptunes $\left(M \sin i=3-30 M_{\oplus}\right.$ and $P<50$ days), H10 found an occurrence rate of $15_{-4}^{+5} \%$

\footnotetext{
* Based on observations obtained at the W. M. Keck Observatory, which is operated jointly by the University of California and the California Institute of Technology. Keck time has been granted by both NASA and the University of California.

10 http://exoplanets.org
}

with a $24 \%$ upper limit (95\% confidence). This is consistent with results from the Anglo-Australian Telescope (O'Toole et al. 2009)-two detected planets in the mass range $M \sin i=$ 5-30 $M_{\oplus}$ with $P<16$ days orbiting 24 FGK dwarfs. The initial estimate of an occurrence rate of $30 \% \pm 10 \%$ for $M \sin i=3-30 M_{\oplus}$ and $P<50$ days based on measurements with HARPS (Mayor et al. 2009) is consistent with a close-in planet mass function that rises steeply with decreasing planet mass, but is inconsistent with the $\mathrm{H} 10$ occurrence measurement.

Population synthesis models of planet formation (Ida \& Lin 2004, 2008; Mordasini et al. 2009) predict a dearth of close-in, low-mass planets. This "desert" emerges in the simulations from fast migration and accelerating planet growth. Most planets are born near or beyond the ice line and those that grow to a critical mass of several Earth masses either rapidly spiral inward to the host star or undergo runaway gas accretion and become massive gas giants. The distribution of planets in $\mathrm{H} 10$ contradicts this prediction; the highest planet occurrence rate is in the 3-30 $M_{\oplus}$ regime with short orbital periods. The two planets reported here have orbital parameters that place them in the so-called desert.

To measure the planet occurrence rate as a function of planet mass, H10 included previously detected planets as well as unannounced "planet candidates." Including candidates was necessary to reliably estimate occurrence fractions for lowmass planets, even though the candidates had formal false alarm probabilities (FAPs) as large as 5\% at the time of the analysis (2010 June). Such an FAP implies that the planet is very likely to exist, but it is too high for the secure announcement of a definite planet detection with well-measured orbital parameters. Since then, we continued to intensively observe the planet candidates. Based on the new confirmatory data we report two of them here as bona fide planets. We present HD $97658 \mathrm{~b}$, a closein, super-Earth planet identified as "Candidate 3 " in Howard et al. (2010b), and Gl 785 b, a Neptune-mass planet identified as "Candidate 7." 
Table 1

Stellar Properties of HD 97658 and G1 785

\begin{tabular}{|c|c|c|c|}
\hline Parameter & HD 97658 & Gl 785 & Source \\
\hline Spectral type & K1 V & $\mathrm{K} 2 \mathrm{~V}$ & Gray et al. (2003) \\
\hline$M_{V}$ & $6.27 \pm 0.10$ & $6.13 \pm 0.02$ & Tycho + Hipparcos \\
\hline$B-V$ & $0.843 \pm 0.022$ & $0.906 \pm 0.017$ & Tycho \\
\hline$V$ & $7.762 \pm 0.012$ & $5.723 \pm 0.009$ & Tycho \\
\hline$J$ & $6.203 \pm 0.023$ & $4.112 \pm 0.294$ & 2MASS \\
\hline$H$ & $5.821 \pm 0.017$ & $3.582 \pm 0.266$ & 2MASS \\
\hline$K$ & $5.734 \pm 0.018$ & $3.501 \pm 0.232$ & 2MASS \\
\hline Distance (pc) & $21.11 \pm 0.33$ & $8.911 \pm 0.024$ & Hipparcos \\
\hline$T_{\text {eff }}(\mathrm{K})$ & $5170 \pm 44$ & $5144 \pm 50$ & $\mathrm{SME}^{\mathrm{a}}$ \\
\hline $\log g$ & $4.63 \pm 0.06$ & $4.60 \pm 0.06$ & SME \\
\hline$[\mathrm{Fe} / \mathrm{H}]$ & $-0.23 \pm 0.03$ & $+0.08 \pm 0.03$ & SME \\
\hline$v \sin i\left(\mathrm{~km} \mathrm{~s}^{-1}\right)$ & $0.5 \pm 0.5$ & $0.5 \pm 0.5$ & SME \\
\hline$L_{\star}\left(L_{\odot}\right)$ & $0.30 \pm 0.02$ & $0.34 \pm 0.02$ & $\mathrm{Y}^{2}+\mathrm{SME}^{\mathrm{b}}$ \\
\hline$M_{\star}\left(M_{\odot}\right)$ & $0.78 \pm 0.02$ & $0.85 \pm 0.02$ & $\mathrm{Y}^{2}+\mathrm{SME}$ \\
\hline$R_{\star}\left(R_{\odot}\right)$ & $0.68 \pm 0.02$ & $0.73 \pm 0.02$ & $\mathrm{Y}^{2}+\mathrm{SME}$ \\
\hline $\log R_{\mathrm{HK}}^{\prime}$ & -4.95 to -5.00 & -4.90 to -5.02 & Isaacson \& Fischer (2010) \\
\hline$S_{\mathrm{HK}}$ & 0.169 to 0.197 & 0.169 to 0.226 & Isaacson \& Fischer (2010) \\
\hline
\end{tabular}

Notes.

a SME: "Spectroscopy Made Easy" package for the analysis of high-resolution spectra. These parameters rely primarily on SME, but have a small dependence also on an iterative analysis incorporating an isochrone search, as described in Valenti et al. (2009).

b $\mathrm{Y}^{2}+\mathrm{SME}$ : based on the Yonsei-Yale isochrones with iterative refinements from SME.

Below we describe the host stars (Section 2) and the RV measurements (Section 3). We analyze these measurements with Keplerian models and assess the probability of spurious detections by computing FAPs (Sections 4 and 5). We describe photometric observations of HD 97658 and the limits they impose on planetary transits (Section 6). We discuss the radii of these planets and a trend in the host star metallicities among low-mass planets (Section 7).

\section{STELLAR PROPERTIES}

We used Spectroscopy Made Easy (Valenti \& Piskunov 1996) to fit high-resolution spectra of HD 97658 (HIP 54906, GJ 3651) and Gl 785 (HD 192310, HIP 99825), using the wavelength intervals, line data, and methodology of Valenti \& Fischer (2005). We further constrained surface gravity using Yonsei-Yale $\left(\mathrm{Y}^{2}\right)$ stellar structure models (Demarque et al. 2004) and revised Hipparcos parallaxes (van Leeuwen 2007), using the iterative method of Valenti et al. (2009). The resulting stellar parameters listed in Table 1 are effective temperature, surface gravity, iron abundance, projected rotational velocity, mass, radius, and luminosity. For reference, Table 1 also lists spectral types, distances derived from Hipparcos, optical photometry from Tycho (Perryman et al. 1997; Bessell 2000), and near infrared photometry from the Two Micron All Sky Survey (2MASS; Cutri et al. 2003). Both stars are early K dwarfs on the main sequence.

HD 97658 lies 0.46 mag below the Hipparcos average main sequence $\left(M_{V}\right.$ versus $\left.B-V\right)$ as defined by Wright (2005). This location is consistent with the low metallicity of $[\mathrm{Fe} / \mathrm{H}]=-0.23 \pm 0.03$. Gl 785 is $0.06 \mathrm{mag}$ above the Hipparcos average main sequence, consistent with its slightly super-solar metallicity of $[\mathrm{Fe} / \mathrm{H}]=+0.08 \pm 0.03$.

Measurements of the cores of the $\mathrm{Ca}$ II $\mathrm{H}$ and $\mathrm{K}$ lines of each spectrum (Figure 1) show low levels of chromospheric activity, as quantified by the $S_{\mathrm{HK}}$ and $\log R_{\mathrm{HK}}^{\prime}$. These chromospheric

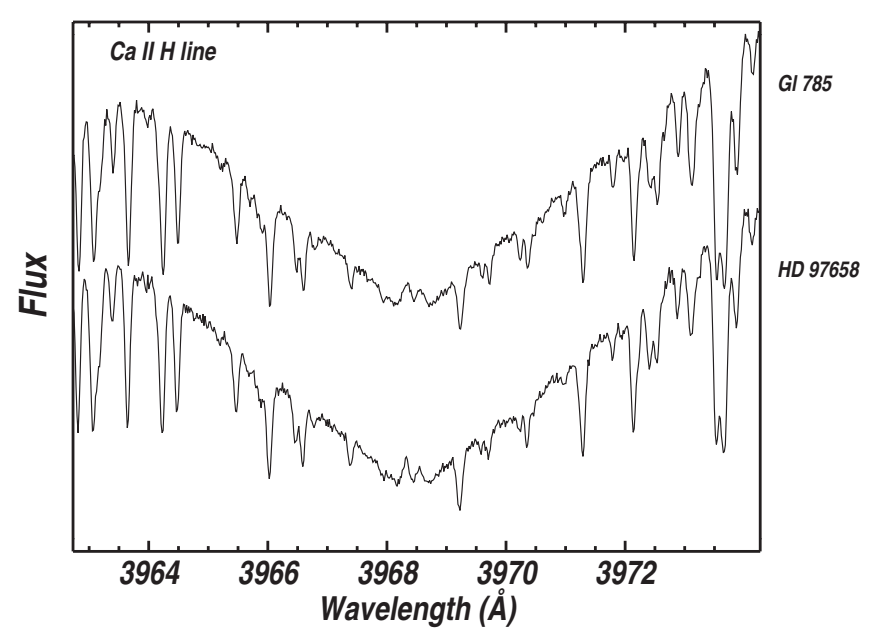

Figure 1. Keck-HIRES spectra of the Ca II H line of the early $\mathrm{K}$ dwarf stars HD 97658 and G1 785. Slight line core emission near $3968 \AA$ indicates modest chromospheric activity.

indices show long-term trends over the six years of measurements, possibly partial activity cycles, so we list ranges of activity indices in Table 1 . We detect a weak correlation between individual RVs and $S_{\mathrm{HK}}$ measurements for HD 97658, but not for Gl 785. This correlation, with a Pearson linear correlation coefficient of $r=+0.35$, does not appear to affect the Keplerian fit of HD 97658 b because the $S_{\mathrm{HK}}$ time series has negligible Fourier power at or near the adopted orbital period, even when the long-term activity trend is removed.

Following Isaacson \& Fischer (2010) and based on $S_{\mathrm{HK}}$, $M_{V}$, and $B-V$, we estimate an RV jitter of $1.5 \mathrm{~m} \mathrm{~s}^{-1}$ for these stars. This empirical estimate is based on an ensemble of stars with similar characteristics and accounts for RV variability due to rotational modulation of stellar surface features, stellar pulsation, undetected planets, and uncorrected systematic errors in the velocity reduction (Saar et al. 1998; Wright 2005). Jitter is added in quadrature to the RV measurement uncertainties for Keplerian modeling.

\section{KECK-HIRES VELOCITY MEASUREMENTS}

We observed HD 97658 and Gl 785 with the HIRES echelle spectrometer (Vogt et al. 1994) on the 10 m Keck I telescope. The observations of each star span six years (2004-2010). All observations were made with an iodine cell mounted directly in front of the spectrometer entrance slit. The dense set of molecular absorption lines imprinted on the stellar spectra provides a robust wavelength fiducial against which Doppler shifts are measured, as well as strong constraints on the shape of the spectrometer instrumental profile at the time of each observation (Marcy \& Butler 1992; Valenti et al. 1995).

We measured the Doppler shift of each star-times-iodine spectrum using a modeling procedure descended from Butler et al. (1996) as described in Howard et al. (2011). The velocity and corresponding uncertainty for each observation is based on separate measurements for $\sim 700$ spectral chunks each $2 \AA$ wide. Once the two planets announced here emerged as candidates (about two years ago) we increased the nightly cadence of measurements and made three consecutive observations per night to reduce the Poisson noise from photon statistics. We calculate one mean velocity for multiple observations in a $2 \mathrm{hr}$ interval.

The highest RV measurement precision using Keck-HIRES has been achieved on chromospherically inactive late $\mathrm{G}$ and 


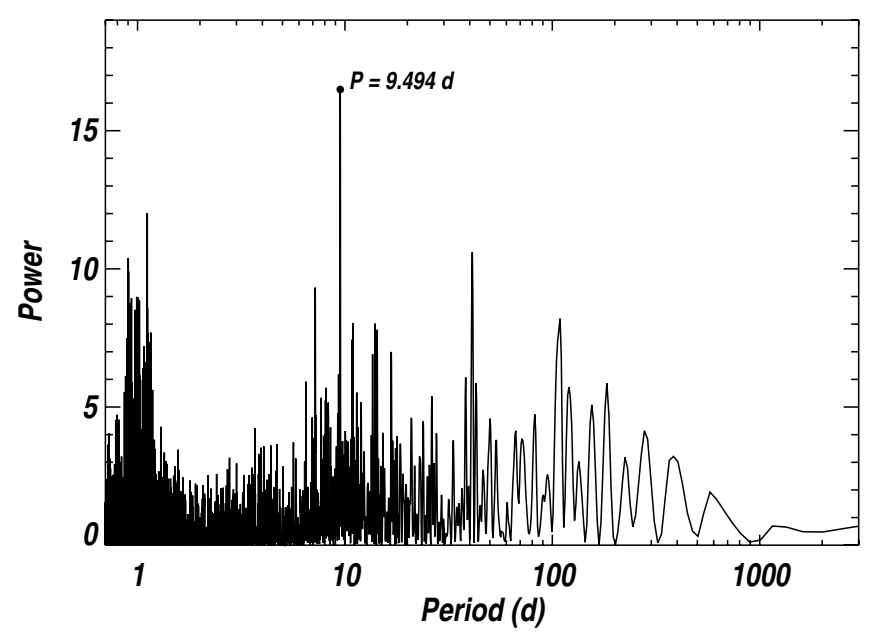

Figure 2. Lomb-Scargle periodogram of RV measurements of HD 97658. The tall peak near $P=9.494$ days suggests a planet with that orbital period.

early $\mathrm{K}$ dwarfs, like the two stars presented here. The quietest of these stars are stable over many years at the $\sim 1.5-2.0 \mathrm{~m} \mathrm{~s}^{-1}$ level (Howard et al. 2009, 2010a, 2011); velocity residuals are due to astrophysical perturbations, instrumental/systematic errors, and Poisson noise. All of the measurements reported here were made after the HIRES CCD upgrade in 2004 August and do not suffer from the higher noise and systematic errors that limited the precision of pre-upgrade measurements to $\sim 2-3 \mathrm{~m} \mathrm{~s}^{-1}$ for most stars.

For each star we constructed a single-planet Keplerian model using the orbit fitting techniques described in Howard et al. (2010a) and the partially linearized, least-squares fitting procedure described in Wright \& Howard (2009). The Keplerian parameter uncertainties for each planet were derived using a Monte Carlo method (Marcy et al. 2005) and do not account for correlations between parameter errors. Uncertainties in $M \sin i$ reflect uncertainties in $M_{\star}$ and the orbital parameters.

\section{HD 97658}

The RVs and $S_{\mathrm{HK}}$ values from Keck-HIRES are listed in Table 2. Figure 2 shows a Lomb-Scargle periodogram (Lomb 1976; Scargle 1982) of the RVs with a substantial peak at 9.494 days. We used that period, as well as a wide variety of other trial periods, as seeds for the Keplerian fitting algorithm (Wright \& Howard 2009). Our search identified as the best fit the single-planet orbital solution listed in Table 3 and depicted in as a time series in Figure 3.

We also tried fitting the RVs with an eccentric Keplerian model and found a best-fit solution with a nearly identical orbital period and $e=0.17 \pm 0.17$, which is consistent with circular at the $1 \sigma$ level. The detection of nonzero eccentricity with better than $95 \%$ confidence $(2 \sigma)$ requires approximately $e / \sigma_{e}>2.45$, where $\sigma_{e}=\sigma / K \cdot(2 / N)^{0.5}, \sigma$ is the measurement uncertainty (including jitter) and $N$ is the number of uniformly phase-distributed measurements (Valenti et al. 2009; Lucy \& Sweeney 1971). Our measurements do not meet this criterion. Furthermore, the eccentric model does not improve $\sqrt{\chi_{v}^{2}}$ compared to the circular model. We adopt the circular orbit model in Table 3.

We considered the null hypothesis - that the observed RVs are the chance arrangement of random velocities masquerading as a coherent signal-by calculating two FAPs. Using the
Table 2

Radial Velocities and $S_{\text {HK }}$ values for HD 97658

\begin{tabular}{|c|c|c|c|}
\hline JD - 2440000 & $\begin{array}{c}\text { Radial Velocity } \\
\left(\mathrm{m} \mathrm{s}^{-1}\right)\end{array}$ & $\begin{array}{c}\text { Uncertainty } \\
\left(\mathrm{m} \mathrm{s}^{-1}\right)\end{array}$ & $S_{\mathrm{HK}}$ \\
\hline 13398.04143 & 3.40 & 0.78 & 0.197 \\
\hline 13748.03543 & 1.41 & 0.79 & 0.190 \\
\hline 13806.96152 & 3.56 & 0.79 & 0.187 \\
\hline 14085.15873 & -2.56 & 0.83 & 0.178 \\
\hline 14246.87902 & -3.08 & 0.73 & 0.176 \\
\hline 14247.83980 & -5.21 & 1.07 & 0.175 \\
\hline 14248.94470 & -0.60 & 1.16 & 0.169 \\
\hline 14249.80244 & 1.19 & 1.24 & 0.174 \\
\hline 14250.83983 & -0.72 & 0.99 & 0.174 \\
\hline 14251.89455 & 0.64 & 1.09 & 0.172 \\
\hline 14255.87104 & -1.07 & 0.79 & 0.174 \\
\hline 14277.81740 & -1.94 & 1.04 & 0.177 \\
\hline 14278.83838 & 1.33 & 1.03 & 0.175 \\
\hline 14279.83000 & 1.07 & 1.00 & 0.176 \\
\hline 14294.76351 & -0.07 & 1.15 & 0.169 \\
\hline 14300.74175 & 3.77 & 1.22 & 0.172 \\
\hline 14304.76223 & -2.53 & 1.23 & 0.174 \\
\hline 14305.75910 & -0.11 & 0.81 & 0.174 \\
\hline 14306.77175 & 3.55 & 1.15 & 0.169 \\
\hline 14307.74725 & 4.39 & 0.83 & 0.175 \\
\hline 14308.75077 & 6.43 & 0.84 & 0.176 \\
\hline 14309.74773 & 5.28 & 1.23 & 0.176 \\
\hline 14310.74343 & 4.32 & 1.20 & 0.175 \\
\hline 14311.74391 & 7.30 & 1.15 & 0.176 \\
\hline 14312.74242 & -0.26 & 1.18 & 0.177 \\
\hline 14313.74419 & -1.57 & 1.26 & 0.178 \\
\hline 14314.75074 & 2.20 & 1.22 & 0.174 \\
\hline 14455.15432 & -5.71 & 1.18 & 0.182 \\
\hline 14635.79759 & -1.70 & 1.09 & 0.175 \\
\hline 14780.12544 & -4.63 & 1.22 & 0.177 \\
\hline 14807.09051 & -2.07 & 1.26 & 0.173 \\
\hline 14808.15781 & 2.09 & 1.30 & 0.171 \\
\hline 14809.14349 & 2.48 & 1.16 & 0.173 \\
\hline 14810.02507 & 8.16 & 1.29 & 0.173 \\
\hline 14811.11469 & 2.77 & 1.28 & 0.173 \\
\hline 14847.11818 & -0.50 & 1.40 & 0.172 \\
\hline 14927.89832 & 3.45 & 1.37 & 0.170 \\
\hline 14928.96319 & -2.78 & 1.30 & 0.170 \\
\hline 14929.84171 & -3.59 & 1.22 & 0.169 \\
\hline 14954.97010 & 1.71 & 1.13 & 0.171 \\
\hline 14955.92258 & 2.61 & 0.59 & 0.172 \\
\hline 14956.90564 & 3.79 & 0.64 & 0.172 \\
\hline 14963.96612 & 4.04 & 0.66 & 0.169 \\
\hline 14983.87266 & 0.73 & 0.70 & 0.170 \\
\hline 14984.90278 & -0.75 & 0.71 & 0.171 \\
\hline 14985.84542 & -2.55 & 0.69 & 0.171 \\
\hline 14986.88960 & -3.00 & 0.69 & 0.170 \\
\hline 14987.89549 & -4.46 & 0.68 & 0.170 \\
\hline 14988.84400 & -4.65 & 0.66 & 0.170 \\
\hline 15041.75244 & 7.08 & 1.35 & 0.169 \\
\hline 15164.11579 & 4.71 & 1.31 & 0.173 \\
\hline 15188.15802 & -0.91 & 0.76 & 0.170 \\
\hline 15190.13283 & -4.78 & 0.71 & 0.170 \\
\hline 15191.16082 & -1.50 & 0.77 & 0.170 \\
\hline 15192.12820 & 1.97 & 0.69 & 0.171 \\
\hline 15193.11592 & 3.52 & 0.67 & 0.172 \\
\hline 15197.14316 & -0.24 & 0.71 & 0.171 \\
\hline 15198.06394 & -1.62 & 0.73 & 0.172 \\
\hline 15199.08955 & -2.12 & 0.72 & 0.172 \\
\hline 15256.95777 & 3.84 & 0.71 & 0.180 \\
\hline 15285.94217 & -1.43 & 0.68 & 0.175 \\
\hline 15289.83015 & 0.99 & 0.64 & 0.178 \\
\hline 15311.78396 & -4.52 & 0.66 & 0.173 \\
\hline 15312.85958 & -2.93 & 0.62 & 0.173 \\
\hline 15313.76751 & 0.82 & 0.65 & 0.172 \\
\hline
\end{tabular}


Table 2

(Continued)

\begin{tabular}{|c|c|c|c|}
\hline JD - 2440000 & $\begin{array}{l}\text { Radial Velocity } \\
\qquad\left(\mathrm{m} \mathrm{s}^{-1}\right)\end{array}$ & $\begin{array}{l}\text { Uncertainty } \\
\left(\mathrm{m} \mathrm{s}^{-1}\right)\end{array}$ & $S_{\mathrm{HK}}$ \\
\hline 15314.78094 & 3.73 & 0.65 & 0.172 \\
\hline 15317.96407 & 1.70 & 0.65 & 0.174 \\
\hline 15318.94543 & -3.46 & 0.67 & 0.175 \\
\hline 15319.90113 & -4.34 & 0.66 & 0.176 \\
\hline 15320.85915 & -5.55 & 0.57 & 0.180 \\
\hline 15321.83386 & -2.68 & 0.62 & 0.181 \\
\hline 15342.87812 & -1.40 & 0.63 & 0.176 \\
\hline 15343.82903 & -1.37 & 0.67 & 0.176 \\
\hline 15344.88076 & 0.84 & 0.73 & 0.175 \\
\hline 15350.78135 & -4.25 & 0.62 & 0.173 \\
\hline 15351.88526 & -0.04 & 0.63 & 0.174 \\
\hline 15372.75655 & 2.37 & 0.63 & 0.179 \\
\hline 15373.78353 & -0.22 & 0.60 & 0.179 \\
\hline 15374.75786 & -0.32 & 0.61 & 0.178 \\
\hline 15375.77512 & -1.73 & 0.61 & 0.177 \\
\hline 15376.74467 & -1.66 & 0.60 & 0.177 \\
\hline 15377.74062 & -0.77 & 0.59 & 0.177 \\
\hline 15378.74257 & 3.55 & 0.65 & 0.176 \\
\hline 15379.79041 & 0.84 & 0.63 & 0.176 \\
\hline 15380.74378 & 6.24 & 0.60 & 0.175 \\
\hline 15400.74241 & 1.31 & 0.72 & 0.177 \\
\hline 15401.76937 & 2.23 & 1.41 & 0.181 \\
\hline 15403.73903 & -1.12 & 0.74 & 0.176 \\
\hline 15404.73645 & -3.00 & 0.67 & 0.181 \\
\hline 15405.74110 & -3.61 & 0.69 & 0.181 \\
\hline 15406.73695 & -1.93 & 0.68 & 0.182 \\
\hline 15407.75726 & 2.44 & 0.81 & 0.180 \\
\hline 15410.73803 & 3.93 & 0.67 & 0.179 \\
\hline 15411.73488 & 0.95 & 0.71 & 0.178 \\
\hline 15412.73197 & -0.23 & 1.26 & 0.178 \\
\hline 15413.73512 & 4.40 & 0.74 & 0.163 \\
\hline
\end{tabular}

Table 3

Orbital Solution for HD 97658 b

\begin{tabular}{lc}
\hline \hline Parameter & Value \\
\hline$P($ days $)$ & $9.494 \pm 0.005$ \\
$T_{c}^{a}(\mathrm{JD}-2,440,000)$ & $15375.01 \pm 0.64$ \\
$e^{b}$ & $\equiv 0.0$ \\
$K\left(\mathrm{~m} \mathrm{~s}^{-1}\right)$ & $2.75 \pm 0.39$ \\
$M \sin i\left(M_{\oplus}\right)$ & $8.2 \pm 1.2$ \\
$a(\mathrm{AU})$ & $0.0831 \pm 0.0011$ \\
$N_{\text {obs }}($ binned $)$ & 96 \\
Median binned uncertainty $\left(\mathrm{m} \mathrm{s}^{-1}\right)$ & 0.74 \\
Assumed jitter $\left(\mathrm{m} \mathrm{s}^{-1}\right)$ & 1.5 \\
$\sigma\left(\mathrm{m} \mathrm{s}^{-1}\right)$ & 2.78 \\
$\sqrt{\chi_{v}^{2}}$ & 1.59 \\
\hline
\end{tabular}

Notes.

a Time of transit.

b We adopt a circular orbital solution for this planet.

method described in Howard et al. (2010a), we computed the improvement in $\Delta \chi^{2}$ from a constant velocity model to a Keplerian model for $10^{3}$ scrambled data sets. In the first FAP test, we allowed for eccentric single-planet orbital solutions in the scrambled data sets. We found that three scrambled data sets had a larger $\Delta \chi^{2}$ than the measured velocities, implying an FAP of $\sim 0.003$ for this scenario. When we restricted the search for orbital solutions to circular orbits, none of the scrambled data sets had a larger $\Delta \chi^{2}$ than measured velocities, implying an FAP of less than $\sim 0.001$.

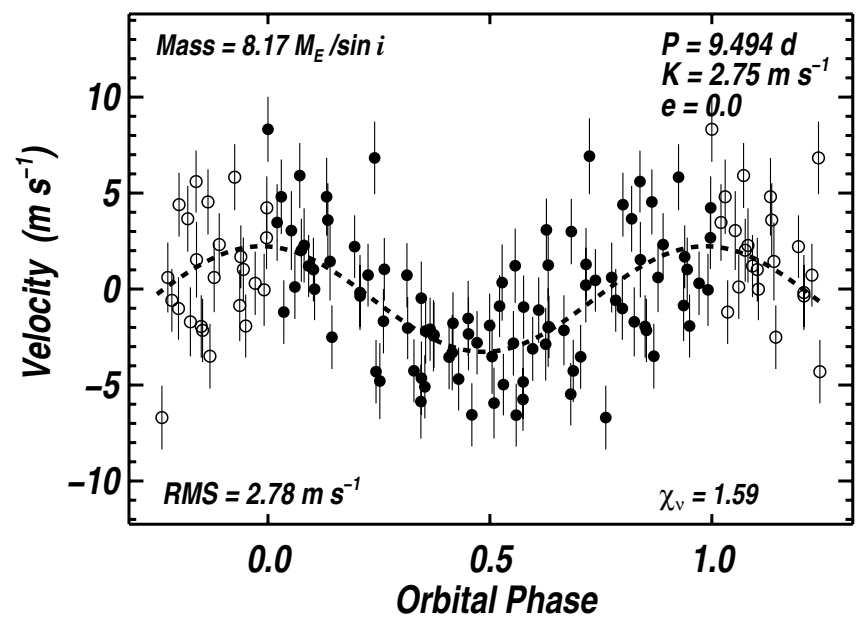

Figure 3. Single-planet model for the RVs of HD 97658, as measured by Keck-HIRES. The dashed line shows the best-fit circular orbital solution. Filled circles represent phased measurements while the open circles represent the same velocities wrapped one orbital phase. The error bars show the quadrature sum of measurement uncertainties and $1.5 \mathrm{~m} \mathrm{~s}^{-1}$ jitter.

The rms of $2.78 \mathrm{~m} \mathrm{~s}^{-1}$ about the single-planet model is relatively high compared to the $1.67 \mathrm{~m} \mathrm{~s}^{-1}$ typical uncertainty (the quadrature sum of the median single measurement error and $1.5 \mathrm{~m} \mathrm{~s}^{-1}$ assumed jitter). This suggests that the measured RVs are compatible with additional detectable planets. We computed a periodogram of the RV residuals to the singleplanet fit and found several periods with considerable power in the range $\sim 40-200$ days. These peaks correspond to Doppler signals with $\sim 1.5-3 \mathrm{~m} \mathrm{~s}^{-1}$ semi-amplitudes. We considered two-planet orbital solutions with $P_{b}$ seeded with the best-fit value from the single-planet model and $P_{c}$ seeded with peaks in the residual periodogram. We allowed all orbital parameters including eccentricities to float in the two-planet fitting process (Wright \& Howard 2009). No two-planet solutions were found with an FAP below 5\%. We will continue to observe this star in search of additional planets.

\section{GL 785}

The RVs and $S_{\mathrm{HK}}$ values from the Keck-HIRES measurements of Gl 785 are listed in Table 4. Figure 4 shows a Lomb-Scargle periodogram (Lomb 1976; Scargle 1982) of the RVs with a substantial peak near 74.4 days. We identify the peaks near 1.0 day as stroboscopic aliases of the sidereal day with the 74.4 days signal and other long periods (Dawson \& Fabrycky 2010). We used 74.4 days, as well as a wide variety of other periods, as seed periods for the single-planet Keplerian fitting algorithm (Wright \& Howard 2009). Our search identified as the best fit the single-planet orbital solution listed in Table 5 and depicted as a time series in Figure 5. The orbital eccentricity of $0.30 \pm 0.09$ is significant at the $3 \sigma$ level.

We considered the null hypothesis for the observed periodic signal in the measured RVs of Gl 785 by computing an FAP using the method described in Section 4, including allowing for eccentric solutions with the scrambled data sets. We found that none of the $10^{3}$ scrambled data sets had a larger $\Delta \chi^{2}$ than the measured velocities, implying an FAP of less than $\sim 0.001$.

With an rms of $2.06 \mathrm{~m} \mathrm{~s}^{-1}$ and a featureless periodogram of velocity residuals to the one-planet model, we do not see evidence for a second detectable planet orbiting Gl 785. 
Table 4

Radial Velocities and $S_{\mathrm{HK}}$ Values for G1 785

\begin{tabular}{|c|c|c|c|}
\hline JD-2440000 & $\begin{array}{l}\text { Radial Velocity } \\
\qquad\left(\mathrm{m} \mathrm{s}^{-1}\right)\end{array}$ & $\begin{array}{l}\text { Uncertainty } \\
\left(\mathrm{m} \mathrm{s}^{-1}\right)\end{array}$ & $S_{\mathrm{HK}}$ \\
\hline 13237.92941 & 1.73 & 0.59 & 0.2103 \\
\hline 13301.71519 & 4.76 & 1.13 & 0.2260 \\
\hline 13549.02705 & -2.75 & 1.02 & 0.2040 \\
\hline 13926.01730 & -1.63 & 0.56 & 0.2023 \\
\hline 13982.83072 & -0.75 & 0.50 & 0.1963 \\
\hline 14247.08230 & -3.60 & 0.67 & 0.1955 \\
\hline 14248.11326 & -5.82 & 0.96 & 0.1950 \\
\hline 14249.12216 & -3.06 & 1.09 & 0.1920 \\
\hline 14252.08848 & 1.73 & 0.93 & 0.1880 \\
\hline 14256.08153 & -0.29 & 0.70 & 0.1890 \\
\hline 14279.03644 & 0.22 & 1.14 & 0.1920 \\
\hline 14280.04184 & 3.05 & 1.05 & 0.1940 \\
\hline 14286.03340 & -4.38 & 1.18 & 0.1910 \\
\hline 14294.99649 & -3.74 & 1.02 & 0.1950 \\
\hline 14634.06380 & 8.57 & 1.15 & 0.1790 \\
\hline 14634.98879 & 4.22 & 1.08 & 0.1830 \\
\hline 14636.03115 & 4.50 & 1.10 & 0.1820 \\
\hline 14637.06862 & 3.17 & 1.12 & 0.1820 \\
\hline 14638.02072 & 3.31 & 1.13 & 0.1830 \\
\hline 14639.05307 & 6.06 & 1.11 & 0.1850 \\
\hline 14640.12929 & 2.63 & 1.08 & 0.1850 \\
\hline 14640.97219 & 6.18 & 1.10 & 0.1850 \\
\hline 14642.09539 & 2.88 & 1.20 & 0.1870 \\
\hline 14644.10213 & 6.65 & 1.23 & 0.1870 \\
\hline 14688.96417 & -2.62 & 1.09 & 0.1820 \\
\hline 14689.98535 & -4.33 & 1.20 & 0.1830 \\
\hline 14723.77286 & 3.43 & 1.19 & 0.1840 \\
\hline 14724.80700 & 7.18 & 1.12 & 0.1830 \\
\hline 14808.68992 & -2.04 & 1.01 & 0.1820 \\
\hline 14984.07717 & -1.89 & 1.19 & 0.1750 \\
\hline 15019.01660 & 1.78 & 1.10 & 0.1740 \\
\hline 15026.96895 & -2.94 & 1.18 & 0.1740 \\
\hline 15042.96436 & 0.91 & 1.13 & 0.1750 \\
\hline 15073.75665 & 0.42 & 0.61 & 0.1760 \\
\hline 15074.75183 & 1.27 & 0.55 & 0.1797 \\
\hline 15077.74110 & 3.29 & 0.64 & 0.1780 \\
\hline 15078.76189 & 1.28 & 0.76 & 0.1770 \\
\hline 15079.73545 & 1.10 & 0.62 & 0.1770 \\
\hline 15080.73918 & 1.01 & 0.58 & 0.1777 \\
\hline 15084.72917 & 1.45 & 0.65 & 0.1743 \\
\hline 15106.75946 & 0.73 & 1.21 & 0.1720 \\
\hline 15109.74590 & -3.42 & 0.71 & 0.1740 \\
\hline 15111.71917 & -3.85 & 0.68 & 0.1753 \\
\hline 15135.74754 & 0.65 & 0.64 & 0.1717 \\
\hline 15169.68272 & 0.91 & 0.72 & 0.1710 \\
\hline 15290.15433 & 0.82 & 0.56 & 0.1687 \\
\hline 15314.13774 & 4.09 & 0.59 & 0.1710 \\
\hline 15319.14050 & 0.05 & 0.70 & 0.1667 \\
\hline 15345.08584 & -5.30 & 0.65 & 0.1720 \\
\hline 15351.09865 & -4.30 & 0.58 & 0.1720 \\
\hline 15352.09190 & -4.02 & 0.61 & 0.1717 \\
\hline 15374.11656 & 0.23 & 0.62 & 0.1743 \\
\hline 15378.11262 & 1.56 & 0.59 & 0.1740 \\
\hline 15379.10643 & -0.50 & 0.62 & 0.1697 \\
\hline 15381.09845 & 3.29 & 0.63 & 0.1717 \\
\hline 15397.04238 & -2.69 & 0.60 & 0.1720 \\
\hline 15400.11504 & -1.87 & 0.61 & 0.1725 \\
\hline 15401.04500 & -1.91 & 0.65 & 0.1717 \\
\hline 15402.08245 & -1.58 & 0.69 & 0.1710 \\
\hline 15404.84477 & -1.96 & 0.58 & 0.1727 \\
\hline 15405.08736 & -0.55 & 0.64 & 0.1710 \\
\hline 15407.93295 & -0.73 & 0.61 & 0.1753 \\
\hline 15412.01241 & -5.21 & 0.60 & 0.1730 \\
\hline 15413.05434 & -4.88 & 0.61 & 0.1740 \\
\hline 15414.04948 & -4.98 & 0.65 & 0.1717 \\
\hline
\end{tabular}

Table 4

(Continued)

\begin{tabular}{lccc}
\hline \hline JD-2440000 & $\begin{array}{c}\text { Radial Velocity } \\
\left(\mathrm{m} \mathrm{s}^{-1}\right)\end{array}$ & $\begin{array}{c}\text { Uncertainty } \\
\left(\mathrm{m} \mathrm{s}^{-1}\right)\end{array}$ & $S_{\mathrm{HK}}$ \\
\hline 15414.92114 & -5.45 & 0.58 & 0.1740 \\
15426.03531 & -2.65 & 0.68 & 0.1717 \\
15427.00892 & -2.32 & 0.62 & 0.1710 \\
15433.99704 & -3.37 & 0.58 & 0.1737 \\
15435.78071 & -2.41 & 0.63 & 0.1756 \\
15436.75896 & -2.30 & 0.63 & $\ldots$ \\
15437.76291 & -3.55 & 0.57 & 0.1747 \\
15438.76140 & -2.62 & 0.59 & 0.1770 \\
15440.75917 & -4.52 & 0.59 & 0.1773 \\
15455.73811 & 1.95 & 0.63 & 0.1750 \\
\hline
\end{tabular}

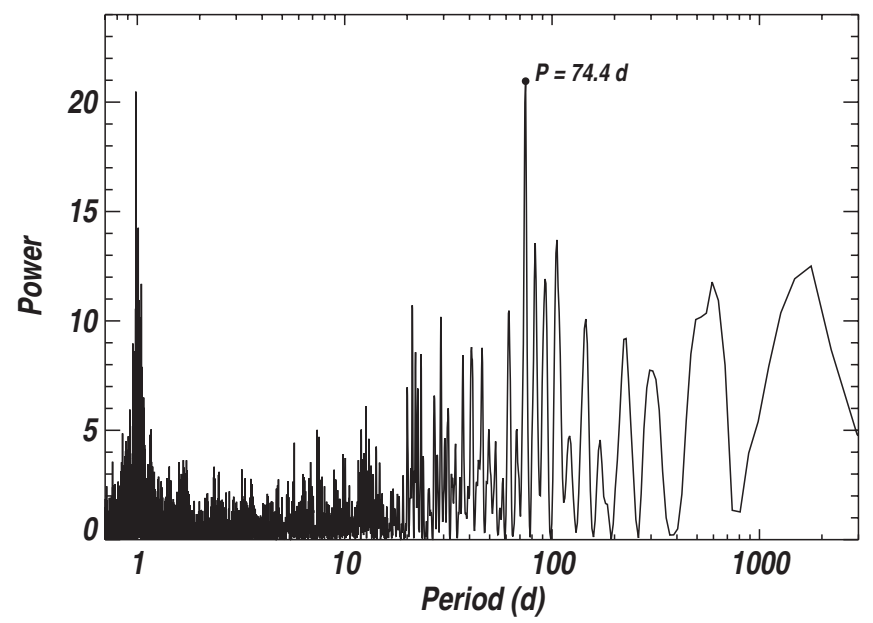

Figure 4. Lomb-Scargle periodogram of RV measurements of Gl 785. The tall peak near $P=74.4$ days suggests a planet with that orbital period.

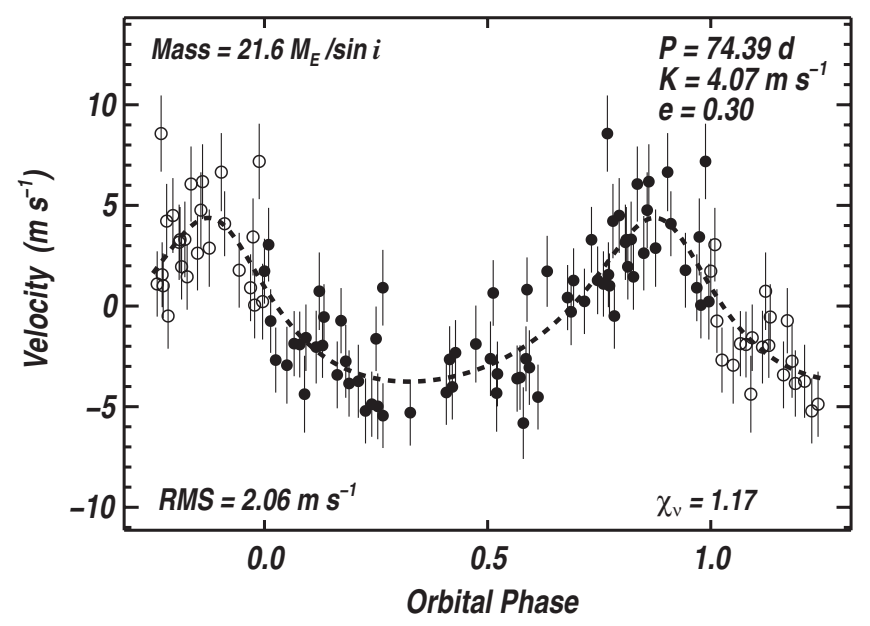

Figure 5. Single-planet model for the RVs of G1 785, as measured by KeckHIRES. The dashed line shows the best-fit eccentric orbital solution. Symbols have the same meanings as in Figure 3.

\section{PHOTOMETRIC OBSERVATIONS}

We acquired photometric observations of HD 97658 with the T12 $0.80 \mathrm{~m}$ Automatic Photometric Telescope (APT), one of several automatic telescopes operated by Tennessee State University at Fairborn Observatory (Eaton et al. 2003). Gl 785 is too far south for this observatory. The APTs can detect short-term, low-amplitude brightness changes in solartype stars resulting from rotational modulation in the visibility 
Table 5

Orbital Solution for G1 785 b

\begin{tabular}{lc}
\hline \hline Parameter & Value \\
\hline$P($ days $)$ & $74.39 \pm 0.12$ \\
$T_{c}^{a}(\mathrm{JD}-2,440,000)$ & $15173.2 \pm 2.0$ \\
$T_{p}^{b}(\mathrm{JD}-2,440,000)$ & $15164.3 \pm 3.6$ \\
$e$ & $0.30 \pm 0.09$ \\
$K\left(\mathrm{~m} \mathrm{~s}^{-1}\right)$ & $4.07 \pm 0.41$ \\
$\omega(\mathrm{deg})$ & $15 \pm 19$ \\
$M$ sin $i\left(M_{\oplus}\right)$ & $21.6 \pm 2.0$ \\
$a(\mathrm{AU})$ & $0.319 \pm 0.005$ \\
$N_{\text {obs }}($ binned $)$ & 73 \\
Median binned uncertainty $\left(\mathrm{m} \mathrm{s}^{-1}\right)$ & 0.68 \\
Assumed jitter $\left(\mathrm{m} \mathrm{s}^{-1}\right)$ & 1.5 \\
$\sigma$ (m s s $\left.^{-1}\right)$ & 2.06 \\
$\sqrt{\chi_{v}^{2}}$ & 1.17 \\
\hline
\end{tabular}

Notes.

a Time of transit.

$\mathrm{b}$ Time of periastron passage.

of active regions, such as starspots and plages (e.g., Henry et al. 1995b) and can also detect longer-term variations produced by the growth and decay of individual active regions and the occurrence of stellar magnetic cycles (e.g., Henry et al. 1995a; Hall et al. 2009). The TSU APTs can disprove the hypothesis that RV variations are caused by stellar activity, rather than planetary reflex motion (e.g., Henry et al. 2000a). Several cases of apparent periodic RV variations in solar-type stars induced by the presence of photospheric starspots have been discussed in the literature (e.g., Queloz et al. 2001; Paulson et al. 2004; Bonfils et al. 2007; Forveille et al. 2009). Photometry of planetary candidate host stars is also useful to search for transits of the planetary companions (e.g., Henry et al. 2000b; Sato et al. 2005; Gillon et al. 2007; Barbieri et al. 2007).

The T12 $0.80 \mathrm{~m}$ APT is equipped with a two-channel photometer that uses two EMI 9124QB bi-alkali photomultiplier tubes to make simultaneous measurements of one star in the Strömgren $b$ and $y$ passbands. The T12 APT is functionally identical to the T8 APT described in Henry (1999).

During three consecutive observing seasons between 2008 January and 2010 June, the APT acquired 318 differential brightness measurements of HD 97658 with respect to the comparison star HD $99518(V=7.71, B-V=0.343, \mathrm{~F} 0)$. We combined the $b$ and $y$ differential magnitudes into $(b+y) / 2$ measurements achieving typical single measurement precision of 1.5-2.0 mmag (Henry 1999). These measurements are plotted in the top panel of Figure 6 and have a standard deviation of 1.99 mmag, consistent with measurement error. Periodogram analysis confirms the absence of periodic variability between 1 and 100 days. The same measurements are plotted modulo the $\mathrm{RV}$ period in the second panel of Figure 6. A least-squares sine fit gives a semi-amplitude of $0.09 \pm 0.14 \mathrm{mmag}$. This tight limit to photometric variability at the RV period supports the hypothesis that the RV signal is due to stellar reflex motion from a planet in motion. Measurements near phase 0.0 are replotted on an expanded scale in the bottom panel of Figure 6 . The precision and phase coverage of our photometry are insufficient to detect shallow transits.

\section{DISCUSSION}

We announce two low-mass planets that were reported as anonymous "planet candidates" in Howard et al. (2010b).
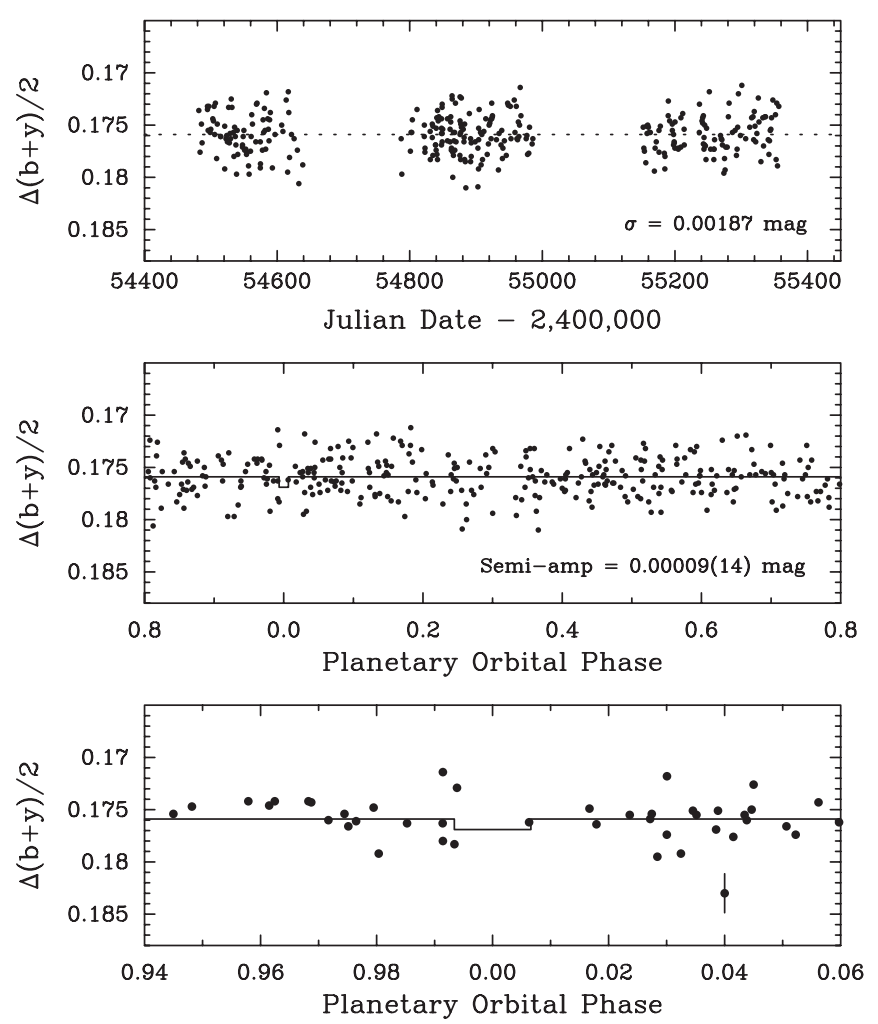

Figure 6. Top panel: the 318 Stromgren $(b+y) / 2$ differential magnitudes of HD 97658 plotted against heliocentric Julian Date. The standard deviation of these normalized observations from their mean (dotted line) is $1.99 \mathrm{mmag}$. The second and third observing seasons have been normalized to match the mean brightness of the first season; the second and third year corrections were 1.75 and 0.70 mmag, respectively. This removes small year-to-year brightness changes in HD 97658 and its comparison star and maximizes sensitivity to brightness variability on night-to-night timescales. Middle panel: the measurements plotted modulo the RV period with phase 0.0 equal to the predicted time of midtransit (Table 3). Bottom panel: the measurements near phase 0.0 plotted on an expanded scale. The vertical error bar in the lower right corresponds to the 1.87 mmag single measurement uncertainty. The duration of a hypothetical central transit is $3 \mathrm{hr}( \pm 0.0066$ phase units); the uncertainty of the transit midpoint is 0.64 days ( 0.067 phase units). The solid curve in the two lower panels approximates the depth $(0.001 \mathrm{mag})$ and duration $(3 \mathrm{hr})$ of a water/ice planet.

HD $97658 \mathrm{~b}$ is a super-Earth planet with minimum mass $M \sin i=8.2 \pm 1.2 M_{\oplus}$ in an orbit of $P=9.494 \pm 0.005$ around a K1 dwarf star. Gl $785 \mathrm{~b}$ is a Neptune-mass planet with minimum mass $M \sin i=21.6 \pm 2.0 M_{\oplus}$ in an orbit of $P$ $=74.39 \pm 0.12$ days, also orbiting a $\mathrm{K} 1$ dwarf.

We see no evidence for transits of HD 97658 b, although our ephemeris and photometric phase coverage preclude detection of all but the deepest transits of a bloated planet. However, given the a priori transit probability of $4 \%$, it is instructive to speculate about the transit signatures of various possible planet compositions. Using the models in Seager et al. (2007), an $8 M_{\oplus}$ planet composed of pure $\mathrm{Fe}, \mathrm{MgSiO}_{3}, \mathrm{H}_{2} \mathrm{O}$, or $\mathrm{H}$ would have radii $R_{\mathrm{pl}}=1.3,1.9,2.4$, and $5.5 R_{\oplus}$, producing transits of depth $0.3,0.6,1.0$, and $5.2 \mathrm{mmag}$, respectively. These homogeneous planet models are oversimplified, but set the scale for admixtures of those ingredients. Transits of planets made of solids and water would have depths of $\sim 0.3-1.0 \mathrm{mmag}$, while transits of a planet with a significant atmosphere could be much deeper.

We have no constraints on transits of Gl 785 b because the host star is too far south for APT observations. The a priori transit probability is $1 \%$. For comparison, we considered the transiting planets HAT-P-11b (Bakos et al. 2010) and HAT-P-26b 
(Hartman et al. 2010), which have masses $26 M_{\oplus}$ and $19 M_{\oplus}$ and radii $4.7 R_{\oplus}$ and $6.3 R_{\oplus}$, respectively. The implied densities, 1.38 and $0.42 \mathrm{~g} \mathrm{~cm}^{-3}$, suggest that these planets have considerable gas fractions. If GL $785 \mathrm{~b}$ has a radius in the range 4.7-6.3 $R_{\oplus}$, equatorial transits will be $4.4-7.8 \mathrm{mmag}$ deep. Such transits would be readily detectable from the ground, but would require a considerable observational campaign given the transit time uncertainty of \pm 2.0 days.

Fischer \& Valenti (2005) showed that the occurrence of giant planets with $K>30 \mathrm{~m} \mathrm{~s}^{-1}$ correlates strongly with $[\mathrm{Fe} / \mathrm{H}]$. This work and other metallicity studies (e.g., Gonzalez 1997; Santos et al. 2004; Johnson et al. 2010) have been interpreted as support for core accretion models of exoplanet formation. However, low-metallicity stars might still be able to form less massive planets. Valenti (2010) noted that stars known to host only planets less massive than Neptune $\left(17 M_{\oplus}\right)$ tend to be metal poor relative to the Sun. HD 97658 $\left([\mathrm{Fe} / \mathrm{H}]=-0.23 \pm 0.03, M \sin i=8.2 \pm 1.2 M_{\oplus}\right)$ and $\mathrm{Gl} 785$ $\left([\mathrm{Fe} / \mathrm{H}]=+0.08 \pm 0.03, M \sin i=21.6 \pm 2.0 M_{\oplus}\right)$ are consistent with this tentative threshold. Before interpreting this physically it is necessary to check for metallicity bias in the subsample of stars around which sub-Neptune mass planets can be detected with current techniques. Further, firmly establishing the apparent anti-correlation between host star metallicity and sub-Neptune mass planet occurrence is best done with a well-controlled sample with uniform detection characteristics, similar to Fischer \& Valenti (2005), or with well-understood detectability, similar to the Eta-Earth Survey.

We thank the many observers who contributed to the measurements reported here. We gratefully acknowledge the efforts and dedication of the Keck Observatory staff, especially Scott Dahm, Hien Tran, and Grant Hill for support of HIRES and Greg Wirth for support of remote observing. We are grateful to the time assignment committees of the University of California, NASA, and NOAO for their generous allocations of observing time. Without their long-term commitment to RV monitoring, these long-period planets would likely remain unknown. We acknowledge R. Paul Butler and S. S. Vogt for many years of contributing to the data presented here. G.W.M. acknowledges NASA grant NNX06AH52G. G.W.H. acknowledges support from NASA, NSF, Tennessee State University, and the State of Tennessee through its Centers of Excellence program. This work made use of the SIMBAD database (operated at CDS, Strasbourg, France), NASA's Astrophysics Data System Bibliographic Services, and the NASA Star and Exoplanet Database (NStED). Finally, the authors wish to extend special thanks to those of Hawai 'ian ancestry on whose sacred mountain of Mauna Kea we are privileged to be guests. Without their generous hospitality, the Keck observations presented herein would not have been possible.

\section{REFERENCES}

Bakos, G. Á., et al. 2010, ApJ, 710, 1724

Barbieri, M., et al. 2007, A\&A, 476, L13

Bessell, M. S. 2000, PASP, 112, 961

Bonfils, X., et al. 2007, A\&A, 474, 293
Borucki, W. J., et al. 2010, ApJ, submitted (arXiv:1006.2799)

Butler, R. P., Marcy, G. W., Williams, E., McCarthy, C., Dosanjh, P., \& Vogt, S. S. 1996, PASP, 108, 500

Charbonneau, D., et al. 2009, Nature, 462, 891

Cutri, R. M., et al. 2003, The IRSA 2MASS All-Sky Point Source Catalog, NASA/IPAC Infrared Science Archive, http://irsa.ipac.caltech.edu/ applications/Gator

Dawson, R. I., \& Fabrycky, D. C. 2010, ApJ, 722, 937

Demarque, P., Woo, J., Kim, Y., \& Yi, S. K. 2004, ApJS, 155, 667

Eaton, J. A., Henry, G. W., \& Fekel, F. C. 2003, in The Future of Small Telescopes in the New Millennium: Vol. 3, Science in the Shadows of Giants, ed T. D. Oswalt (Astrophysics and Space Science Library, Vol. 288; Dordrecht: Kluwer), 189

Fischer, D. A., \& Valenti, J. 2005, ApJ, 622, 1102

Fischer, D. A., et al. 2008, ApJ, 675, 790

Forveille, T., et al. 2009, A\&A, 493, 645

Gillon, M., et al. 2007, A\&A, 472, L13

Gonzalez, G. 1997, MNRAS, 285, 403

Gray, R. O., Corbally, C. J., Garrison, R. F., McFadden, M. T., \& Robinson, P. E. 2003, AJ, 126, 2048

Hall, J. C., Henry, G. W., Lockwood, G. W., Skiff, B. A., \& Saar, S. H. 2009, AJ, 138,312

Hartman, J. D., et al. 2010, ApJ, submitted (arXiv:1010.1008)

Henry, G. W. 1999, PASP, 111, 845

Henry, G. W., Baliunas, S. L., Donahue, R. A., Fekel, F. C., \& Soon, W. 2000a, ApJ, 531, 415

Henry, G. W., Eaton, J. A., Hamer, J., \& Hall, D. S. 1995a, ApJS, 97, 513

Henry, G. W., Fekel, F. C., \& Hall, D. S. 1995b, AJ, 110, 2926

Henry, G. W., Marcy, G. W., Butler, R. P., \& Vogt, S. S. 2000b, ApJ, 529, L41

Howard, A. W., et al. 2009, ApJ, 696, 75

Howard, A. W., et al. 2010a, ApJ, 721, 1467

Howard, A. W., et al. 2010b, Science, 330, 653

Howard, A. W., et al. 2011, ApJ, 726, 73

Ida, S., \& Lin, D. N. C. 2004, ApJ, 604, 388

Ida, S., \& Lin, D. N. C. 2008, ApJ, 685, 584

Isaacson, H., \& Fischer, D. 2010, ApJ, 725, 875

Johnson, J. A., Aller, K. M., Howard, A. W., \& Crepp, J. R. 2010, PASP, 122, 905

Léger, A., et al. 2009, A\&A, 506, 287

Lomb, N. R. 1976, Ap\&SS, 39, 447

Lovis, C., et al. 2006, Nature, 441, 305

Lucy, L. B., \& Sweeney, M. A. 1971, AJ, 76, 544

Marcy, G. W., \& Butler, R. P. 1992, PASP, 104, 270

Marcy, G. W., Butler, R. P., Vogt, S. S., Fischer, D. A., Henry, G. W., Laughlin, G., Wright, J. T., \& Johnson, J. A. 2005, ApJ, 619, 570

Mayor, M., et al. 2009, A\&A, 493, 639

Mordasini, C., Alibert, Y., \& Benz, W. 2009, A\&A, 501, 1139

O'Toole, S., et al. 2009, ApJ, 697, 1263

Paulson, D. B., Saar, S. H., Cochran, W. D., \& Henry, G. W. 2004, AJ, 127, 1644

Perryman, M. A. C., et al. 1997, A\&A, 323, L49

Queloz, D., et al. 2001, A\&A, 379, 279

Saar, S. H., Butler, R. P., \& Marcy, G. W. 1998, ApJ, 498, L153

Santos, N. C., Israelian, G., \& Mayor, M. 2004, A\&A, 415, 1153

Sato, B., et al. 2005, ApJ, 633, 465

Scargle, J. D. 1982, ApJ, 263, 835

Seager, S., Kuchner, M., Hier-Majumder, C. A., \& Militzer, B. 2007, ApJ, 669, 1279

Valenti, J. A. 2010, in IAU Symp. 265, Chemical Abundances in the Universe: Connecting First Stars to Planets, ed. K. Cunha, M. Spite, \& B. Barbuy (Cambridge: Cambridge Univ. Press), 403

Valenti, J. A., Butler, R. P., \& Marcy, G. W. 1995, PASP, 107, 966

Valenti, J. A., \& Fischer, D. A. 2005, ApJS, 159, 141

Valenti, J. A., \& Piskunov, N. 1996, A\&AS, 118, 595

Valenti, J. A., et al. 2009, ApJ, 702, 989

van Leeuwen, F. 2007, A\&A, 474, 653

Vogt, S. S., et al. 1994, Proc. SPIE, 2198, 362

Vogt, S. S., et al. 2010, ApJ, 708, 1366

Wright, J. T. 2005, PASP, 117, 657

Wright, J. T., \& Howard, A. W. 2009, ApJS, 182, 205

Wright, J. T., et al. 2010, PASP, submitted (arXiv:1012.5676) 